\title{
Cooperative Radar Jamming for Groups of Unmanned Air Vehicles
}

\author{
Jongrae Kim \\ Dept. of Engineering \\ University of Leicester \\ Leicester, LE1 7RH, United Kingdom \\ Email: jrk7@le.ac.uk
}

\author{
João P. Hespanha \\ Dept. of Electrical \& Computer Engineering \\ University of California \\ Santa Barbara, CA, 93106-9560, USA \\ Email: hespanhadece.ucsb.edu
}

\begin{abstract}
We consider a scenario where a group of Unmanned Air Vehicles flies in a close formation and cooperates in their use of jamming resources to prevent being tracked by Surface-to-Air Missile (SAM) tracking radars. We consider threats posed by a single or multiple SAMs and investigate their impact on minimum-risk path planning.
\end{abstract}

\section{INTRODUCTION}

Concealment through Electronic Counter-Measures (ECM), more commonly referred to as jamming, consists of transmitting some form of noise to swamp a probing radar signal. Jamming is often used to conceal aircraft from the radars that guide Surface-to-Air Missiles (SAMs).

We consider a scenario in which a group of Unmanned Combat Air Vehicles (UCAVs) uses jamming to avoid threats posed by SAMs. Jamming is typically classified as: self-protection or support depending on whether it is used to protect the aircraft that transmits the ECM noise signal or a different aircraft. Figure 1 shows both forms of jamming: the D-labeled UCAV is engaged in self-protection jamming, whereas the A, B, C UCAVs are engaged in support jamming. Note that the ECM signal transmitted by D can also provide some form of concealment for A, B, C (and vice-versa) however it is must less efficient because it transmits from outside the main lobe of the antenna that is being used to track the other aircraft.

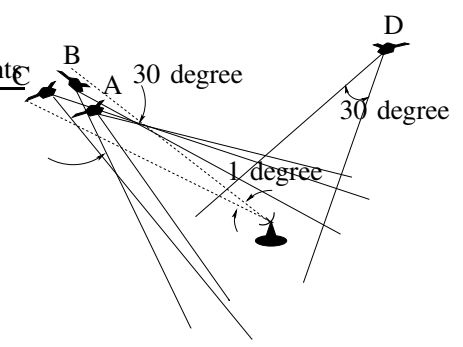

Fig. 1. Cooperative Radar Jamming

This paper is mostly focused on Escort Jamming (EJ), which is a form of support jamming in which aircraft flying

\footnotetext{
This paper is based upon work supported by DARPA under the Space and Naval Warfare Systems Center, San Diego, Contracts Number N66001-01-C-8076 and N66001-04-M-R700. Any opinions, findings, and conclusions or recommendations expressed in this material are those of the authors and do not necessarily reflect the views of DARPA or the Space and Naval Warfare Systems Center, San Diego.
}

in close formation use ECM for mutual concealment. Escort jamming is often used when only one or a few of the aircraft in the formation have ECM transmitters and provide jamming to protect the others. We are mostly interested in situations where several aircraft have ECM transmitters and coordinate their efforts to improve jamming effectiveness by (i) superimposing their signals to increase the range over which jamming is effective against a particular radar (Section II), and/or (ii) directing their transmitters to different radars to conceal the formation from multiple radars (Section III).

Cooperative jamming is investigated from a pathplanning perspective. In particular, we are interested in determining paths for a group of autonomous UCAVs that minimize their risk of being tracked and destroyed by SAM sites. These paths should be selected to make the most efficient use of jamming. We show in Section IV that the computation of minimum-risk paths for UCAVs capable of jamming can be formulated as the weighted anisotropic shortest-path problem considered in [1]. This extends previous work on minimum-risk path planning, which essentially attempted to minimize the Radar Cross Section (RCS) of the UCAVs along the path and did not take jamming into account [1]. Not surprisingly, jamming can have a dramatic effect in reducing risk and in general leads to drastically different minimum-risk paths.

All numerical values for the parameters presented in this paper were taken from the Challenge Problem Version 1.3 set forth by Boeing for the DARPA/IXO program Mixed Initiative Control of Automa-teams (MICA) [2, 3]. These values do not correspond to any real UCAV or SAM radar but permit a realistic testing of jamming effectiveness.

\section{JAMMING EFFECTIVENESS-SINGLE RADAR}

The effectiveness of active (transmitting) ECM devices is a function of the Jamming to Signal ratio (J/S) [3, 4]. When $m$ UCAVs cooperatively jam one radar that is tracking a target UCAV, we have

$$
J / S=\frac{\sum_{i=1}^{m} J_{i}}{S},
$$

where $S$ denotes the signal strength of the target return signal and $J_{i}$ the signal strength of the ECM signal generated by the $i$ th UCAV. In general, jamming is effective when 
$J / S$ exceeds a specific burn-through value $(J / S)_{\text {burn }}$ that depends on the characteristics and signal processing used by the radar.

For a radar system with collocated transmitter and receiver antennas, the target return signal $S$ is given by

$$
S=\frac{k_{S} \sigma\left(\theta_{\mathrm{el}}, \theta_{\mathrm{az}}\right)}{R_{T}^{4}},
$$

where $k_{S}$ denotes a radar-dependent coefficient (which depends on transmitted power, wavelength, and antenna gains); $R_{T}$ the distance from the radar to the target UCAV; and $\sigma(\cdot)$ the Radar Cross Section (RCS) of the target UCAV. The RCS is defined as the ratio between the backscatter power per unit solid angle (in the direction of the radar) and the power density that is intercepted by the target. Generally, the RCS varies significantly with the attitude of the target UCAV with respect to the radar, expressed by the elevation and azimuth angles $\theta_{\mathrm{el}}$ and $\theta_{\mathrm{az}}$, respectively.

The jamming signal strengths can be expressed as

$$
J_{i}=\frac{k_{J i} G_{R J_{i}}}{R_{J i}^{2}}
$$

where $R_{J_{i}}$ denotes the distance from the $i$ th UCAV to the radar, $k_{J_{i}}$ a UCAV-dependent coefficient, and $G_{R J_{i}}$ the radar antenna gain in the direction of the $i$ th UCAV. The antenna gain $G_{R J_{i}}$ depends on whether or not the $i$ th jamming UCAV is aligned with the radar's antenna main lobe as it sweeps the target. When this alignment occurs we have main-lobe jamming, otherwise we have side-lobe jamming. In general, the side-lobe antenna gain is significantly smaller than the main-lobe one and many radars employ some form of side lobe cancellation, making side-lobe jamming very inefficient.

Table I contains values for the parameters above from the MICA challenge problem [2]. Figure 2 shows a graphical representation of the UCAV's RCS as a function of the azimuth angle for small elevation angles (up to 20deg).

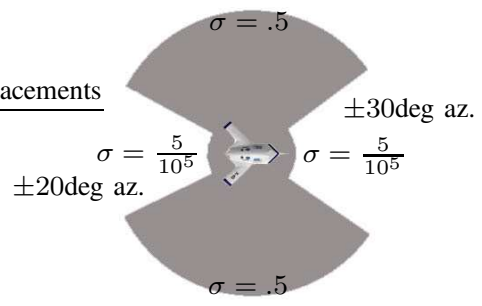

Fig. 2. UCAV's RCS as a function of the azimuth angle $\theta_{\text {az }}$ for elevation angles $\theta_{\mathrm{el}}$ up to 20deg. The RCS is significantly larger for larger elevation angles. Data from the MICA challenge problem version 1.3 [2]

We assume that all UCAVs involved are flying in a close formation, which includes the $m$ jamming UCAVs and the target UCAV. The target UCAV may or may not be one of the jamming UCAVs. In this scenario, we always have main-lobe jamming and all radar-to-UCAV distances are approximately equal so we denote them simply by $R$ :

$$
R \approx R_{T} \approx R_{J_{1}} \approx \cdots \approx R_{J_{m}} .
$$

Combining (1), (2), and (3), we conclude that the burnthrough condition is given by

$$
J / S=\frac{R^{2} \sum_{i=1}^{m} k_{J i} G_{R J_{i}}}{k_{S} \sigma\left(\theta_{\mathrm{el}}, \theta_{\mathrm{az}}\right)} \geq(J / S)_{\mathrm{burn}} .
$$

For a given distance $R$, jamming will succeed as long as the RCS of the target UCAV satisfies

$$
\sigma\left(\theta_{\mathrm{el}}, \theta_{\mathrm{az}}\right) \leq \sigma_{\text {burn }}(R):=\frac{\sum_{i=1}^{m} k_{J i} G_{R J_{i}}}{k_{S}(J / S)_{\text {burn }}} R^{2} .
$$

The right-hand-side of (4) is the maximum target UCAV RCS for which jamming will succeed, which we call the burn-through RCS. Figure 3 shows $\sigma_{\text {burn }}(R)$ as a function of the distance $R$, for several values of the number $m$ of jamming UCAVs.

The path followed by the group of UCAVs has a significant impact on the effectiveness of jamming because there is a strong dependence between the jamming effectiveness and (i) the distance $R$ from the UCAV's to the radar and (ii) the azimuth and elevation angles $\theta_{\mathrm{az}}, \theta_{\mathrm{el}}$ that determine the RCS of the target UCAV. This presents an opportunity to be explored by minimum-risk path planning algorithms. This will be further explored in Section IV.

\section{A. Achievable RCSs}

When the RCS varies significantly according to the attitude of the target UCAV with respect to the radar (as in Figure 2), the UCAV can reduce its RCS by appropriate maneuvering. In practice, the minimum achievable RCS determines how close the target UCAV can approach the radar until jamming becomes ineffective.

From a path planning perspective, three values of the RCS are relevant: The maximum RCS for any orientation of the target UCAV

$$
\sigma_{\max }:=\max _{\theta_{\mathrm{az}} \in[-\pi, \pi]} \max _{\theta_{\mathrm{el}} \in\left[-\frac{\pi}{2}, \frac{\pi}{2}\right]} \sigma\left(\theta_{\mathrm{el}}, \theta_{\mathrm{az}}\right) ;
$$

the maximum RCS over all azimuth angles but for the mostfavorable elevation angle

$$
\sigma_{\mathrm{az}}(R):=\max _{\theta_{\mathrm{az}} \in[-\pi, \pi]} \min _{\theta_{\mathrm{el}} \geq \theta_{\mathrm{el}}^{\min }(R)} \sigma\left(\theta_{\mathrm{el}}, \theta_{\mathrm{az}}\right) ;
$$

and the minimum RCS for any azimuth and elevation angle

$$
\sigma_{\min }(R):=\min _{\theta_{\mathrm{az}} \in[-\pi, \pi]} \min _{\theta_{\mathrm{el}} \geq \theta_{\mathrm{el}}^{\min }(R)} \sigma\left(\theta_{\mathrm{el}}, \theta_{\mathrm{az}}\right),
$$

where $\theta_{\mathrm{el}}^{\min }(R)$ denotes the minimum possible elevation angle. When $\sigma_{\text {burn }}(R) \geq \sigma_{\max }$ the target UCAV can fly with any orientation and remain protected by jamming; when $\sigma_{\text {burn }}(R) \geq \sigma_{\mathrm{az}}(R)$ it can approach the radar from any direction (azimuth), provided that it chooses the most favorable elevation; when $\sigma_{\text {burn }}(R) \geq \sigma_{\min }(R)$ it can only 
TABLE I

UCAV AND SAM-RADAR PARAMETERS FROM THE MICA CHALLENGE PROBLEM VERSION 1.3 [2]

\begin{tabular}{c||c|c|c|c|c}
\hline $\begin{array}{c}\text { UCAV } \\
\text { type }\end{array}$ & $\begin{array}{c}\text { jam. } \\
\text { beam }\end{array}$ & $k_{J}$ & $\begin{array}{c}v_{\min } \min . \\
\text { velocity }\end{array}$ & $\begin{array}{c}d_{\max } \max . \\
\text { desc. rate }\end{array}$ & $\begin{array}{c}\alpha_{\text {att }} \text { ang. } \\
\text { of attack }\end{array}$ \\
\hline \hline small & $30^{\circ}$ & 0.25 & $77 \mathrm{~m} / \mathrm{s}$ & $20 \mathrm{~m} / \mathrm{s}$ & 0 \\
large & $30^{\circ}$ & 1 & $77 \mathrm{~m} / \mathrm{s}$ & $20 \mathrm{~m} / \mathrm{s}$ & 0 \\
\hline
\end{tabular}

(a) UCAV parameters

\begin{tabular}{c||c|c|c|c|c}
\hline $\begin{array}{c}\text { SAM } \\
\text { type }\end{array}$ & $k_{S}$ & $\left(\frac{J}{S}\right)_{\text {burn }}$ & $\begin{array}{c}\text { main-lobe } \\
\text { width }\end{array}$ & $\begin{array}{c}G_{R J} \\
\text { main-lobe }\end{array}$ & $\begin{array}{c}G_{R J} \\
\text { side-lobe }\end{array}$ \\
\hline \hline short-range & $210^{7}$ & 1 & $1^{\circ}$ & 1 & .001 \\
med.-range & $210^{8}$ & 1 & $1^{\circ}$ & 1 & .001 \\
long-range & $210^{9}$ & 1 & $1^{\circ}$ & 1 & .001 \\
\hline
\end{tabular}

(b) SAM tracking-radar parameters

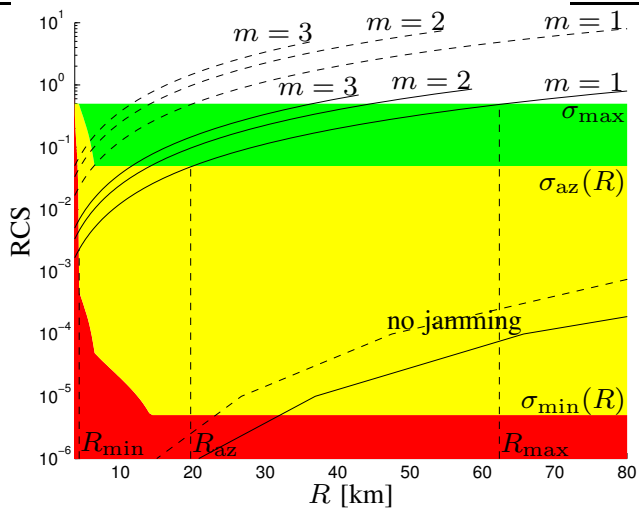

(a) $h_{\min }=3.66 \mathrm{~km}$

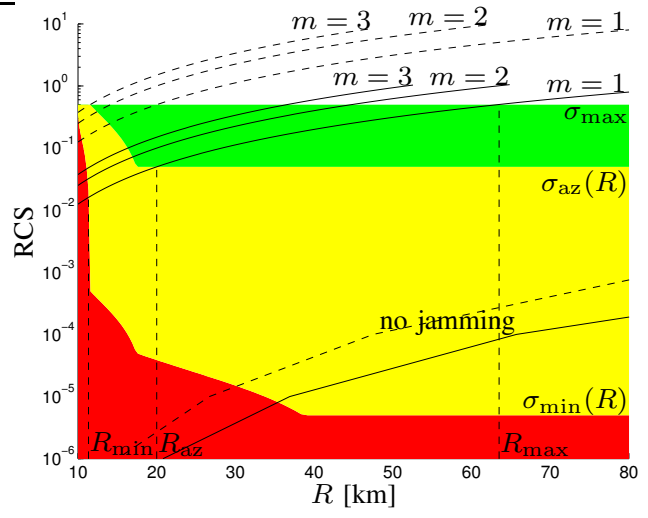

(b) $h_{\min }=10 \mathrm{~km}$

Fig. 3. The six lines at the top of each plot represent $\sigma_{\text {burn }}(R)$ for $m$ small jamming UCAVs, as a function of the distance $R$ to the radar. The two lines at the bottom represent the maximum RCS of the target UCAV for which it will be tracked with probability smaller than $1 \%$, without jamming (cf. Remark 1). The solid lines correspond to a long-range SAM whereas the dashed lines to a medium-range SAM. The four areas defined by the background color correspond (from bottom to top) to RCSs that (i) can never be achieved, (ii) can be achieved by appropriate selection of both the azimuth and elevation angles, (iii) can be achieved for any azimuth angle by appropriate selection of the elevation angle, and (iv) can be achieved for every azimuth and elevation angles. The two plots differ in terms of the assumed minimum altitude $h_{\text {min }}$ allowed for the target UCAV. In the left plot $h_{\mathrm{min}}=3.66 \mathrm{~km}$ is just above the engagement zone for the anti-aircraft artillery (AAA) in [2]. All parameters from Table I and Figure 2.

avoid tracking for the most favorable orientations; when $\sigma_{\text {burn }}(R)<\sigma_{\min }(R)$ jamming will never succeed.

The values of $\sigma_{\mathrm{az}}(R)$ and $\sigma_{\min }(R)$ generally depend on the distance $R$ between the target UCAV and the radar because in choosing the most favorable elevation one is constrained by the UCAV's maximum descent rate. To understand how, we use the following equations:

$$
\sin \left(\theta_{\mathrm{el}}+\theta_{\mathrm{pitch}}\right)=\frac{h}{R}, \quad \sin \left(\theta_{\mathrm{pitch}}+\alpha_{\mathrm{att}}\right)=\frac{d}{v},
$$

where $h$ denotes the UCAV's altitude, $R$ its distance to the radar, $v$ its velocity, $d$ its descent rate, $\theta_{\text {pitch }}$ the pitch angle with respect to the horizontal plane, and $\alpha_{\text {att }}$ the angle of attack (cf. Figure 4). Therefore

$$
\theta_{\mathrm{el}}=\arcsin \frac{h}{R}-\theta_{\mathrm{pitch}}=\arcsin \frac{h}{R}-\arcsin \frac{d}{v}+\alpha_{\mathrm{att}} \text {. }
$$

Therefore the minimum elevation angle is given by

$$
\theta_{\mathrm{el}}^{\min }(R):=\arcsin \frac{h_{\mathrm{min}}}{R}-\arcsin \frac{d_{\mathrm{max}}}{v_{\min }}+\alpha_{\min },
$$

where $h_{\min }$ denotes the lowest admissible altitude, $d_{\max }$ the maximum descent rate, $v_{\min }$ the minimum speed, and $\alpha_{\min }$

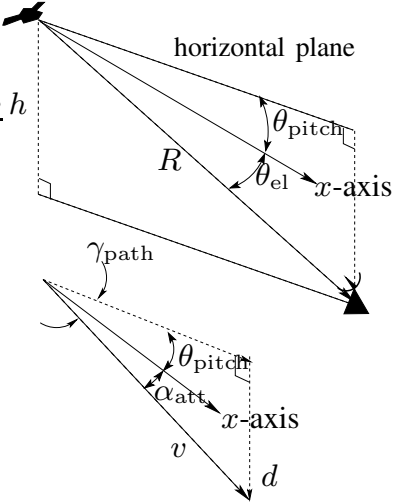

Fig. 4. Computation of the elevation angle. The vector labeled with " $x$-axis" is aligned with the nose of the aircraft (body-frame).

the minimum angle of attack $\alpha_{\min }$. Figure 3 shows $\sigma_{\max }$, $\sigma_{\mathrm{az}}(R)$, and $\sigma_{\min }(R)$ versus the distance $R$.

\section{B. Partition of the airspace}

From the plots in Figure 3, we conclude that there are four distinct radial regions around a radar, which should be 
considered separately by path planning algorithms:

1) The first region $\mathcal{R}_{\text {red }}$ corresponds to distances from the target UCAV to the radar in the interval $\left[0, R_{\min }\right)$, where $R_{\text {min }}$ is obtained by intersecting the curves for $\sigma_{\text {burn }}(R)$ and $\sigma_{\min }(R)$. In this region, jamming will never be effective.

2) The second region $\mathcal{R}_{\text {orange }}$ corresponds to distances from the target UCAV to the radar in the interval $\left[R_{\mathrm{min}}, R_{\mathrm{az}}\right)$, where $R_{\mathrm{az}}$ is obtained by intersecting the curves for $\sigma_{\text {burn }}(R)$ and $\sigma_{\mathrm{az}}(R)$. In this region jamming will only be effective for restricted values of both the azimuth and the elevation angles. Path planning is particularly challenging in this region because it is significantly anisotropic [1].

3) The third region $\mathcal{R}_{\text {yellow }}$ corresponds to distances from the target UCAV to the radar in the interval [ $\left.R_{\mathrm{az}}, R_{\mathrm{max}}\right)$, where $R_{\max }$ is obtained by intersecting the curves for $\sigma_{\text {burn }}(R)$ and $\sigma_{\max }(R)$. In this region jamming can be effective for any direction of motion (azimuth), provided that the elevation is appropriately selected.

4) The forth region $\mathcal{R}_{\text {green }}$ corresponds to distances from the target UCAV to the radar larger than or equal to $R_{\text {max }}$. In this region jamming is always effective regardless of the azimuth and the elevation angles.

The boundaries between these regions depend on the number and type of jamming UCAVs, because $\sigma_{\text {burn }}(R)$ (i.e., the maximum RCS of the target UCAV for which jamming will succeed) also depends on these parameters. These boundaries are highlighted in Figure 3 for a single UCAV jamming a long-range SAM (solid line with the label $m=$ 1 ). From the figure we can see that $\mathcal{R}_{\text {orange }}$ and $\mathcal{R}_{\text {yellow }}$ can be significantly decreased by increasing the number of jamming UCAVs, especially for the long-range SAMs.

Remark 1: Even when none of the UCAVs is actively jamming, the existence of ambient electromagnetic noise may prevent the UCAVs from being tracked if they present very low RCSs to the radar. We show in Figure 3 maximum RCSs for the target UCAV below which it would be tracked with probability lower than $1 \%$, as a function of the distance to the radar. Without jamming, the UCAVs could only fly safely with arbitrary azimuth at distance of roughly $500 \mathrm{~km}$ (not shown in the plots).

\section{MUlTi-RADAR SCENARIO}

We consider now the case in which the group of $m$ UCAVs fly in a region populated by multiple SAM sites, each operated independently and guided by its own tracking radar. We continue to assume that the UCAVs fly in a close formation and cooperate in their use of jamming resources. For concreteness we often refer to the scenario depicted in Figure 5, which was taken from [2]. In this scenario one finds two types of SAM sites: medium- and longrange. Their firing ranges, $40 \mathrm{~km}$ and $80 \mathrm{~km}$, respectively, are indicated by circles centered around the site location. We

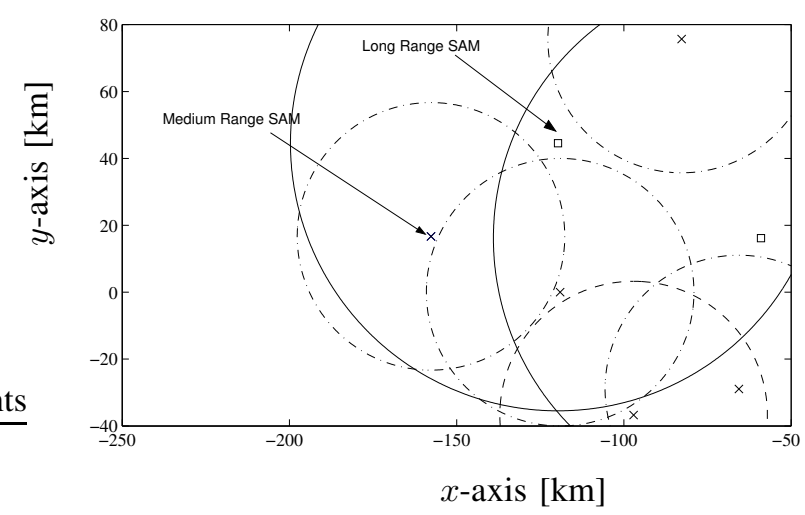

Fig. 5. Battlefield scenario from [2]

assume that the location of each SAM can be estimated with reasonable accuracy, perhaps using the algorithms described in $[5,6]$.

The group of UCAVs can now be threatened simultaneously by multiple SAMs. The partition of the airspace considered in Section II-B needs to be re-defined to take this into account:

1) In the region $\mathcal{R}_{\text {green }}$ jamming is always effective regardless of the azimuth and the elevation angles of the UCAVs. $\mathcal{R}_{\text {green }}$ consists of the locations for which $\forall \theta_{\mathrm{az}} \in[-\pi, \pi], \theta_{\mathrm{el}} \in\left[-\frac{\pi}{2}, \frac{\pi}{2}\right]$,

$$
\sigma_{\text {burn }}^{j} \geq \sigma_{i}^{j}\left(\theta_{\mathrm{el}}, \theta_{\mathrm{az}}\right), \quad \forall i, j,
$$

where the index $j$ ranges over the SAMs within firing range and $i$ ranges over the UCAVs in the group. We denote by $\sigma_{i}^{j}\left(\theta_{\mathrm{el}}, \theta_{\mathrm{az}}\right)$ the RCS that the $i$ th UCAV presents to the $j$ th radar and by $\sigma_{\text {burn }}^{j}$ the burnthrough RCS for the $j$ th radar. When no UCAV is jamming a particular radar $j$, one should take $\sigma_{\text {burn }}^{j}$ to be the maximum RCS for which tracking will fail with high probability (cf. Remark 1).

2) In the region $\mathcal{R}_{\text {yellow }}$ jamming can be effective for any direction of motion (azimuth) for the group, provided that the elevation is appropriately selected. $\mathcal{R}_{\text {yellow }}$ consists of the locations not in the previous region for which $\forall \theta_{\mathrm{az}} \in[-\pi, \pi], \exists \theta_{\mathrm{el}} \geq \theta_{\mathrm{el}}^{\min }(R)$ such that

$$
\sigma_{\text {burn }}^{j} \geq \sigma_{i}^{j}\left(\theta_{\mathrm{el}}, \theta_{\mathrm{az}}\right), \quad \forall i, j .
$$

3) In the region $\mathcal{R}_{\text {orange }}$ jamming will only be effective for restricted values of both the azimuth and the elevation angles for the group. $\mathcal{R}_{\text {orange consists of }}$ the locations not in the previous regions for which $\exists \theta_{\mathrm{az}} \in[-\pi, \pi], \exists \theta_{\mathrm{el}} \geq \theta_{\mathrm{el}}^{\min }(R)$ such that

$$
\sigma_{\text {burn }}^{j} \geq \sigma_{i}^{j}\left(\theta_{\mathrm{el}}, \theta_{\mathrm{az}}\right), \quad \forall i, j .
$$

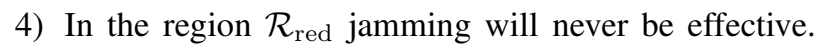
$\mathcal{R}_{\text {red }}$ consists of all locations not in any of the previous regions. 
The above definitions implicitly assume that the UCAVs move along a path, all with similar orientations specified by a single pair of elevation and azimuth angles $\theta_{\mathrm{el}}, \theta_{\mathrm{az}}$.

The satisfaction of the conditions that define these regions depend on the number of UCAVs jamming each radar, since this affects the value of $\sigma_{\text {burn }}^{j}(R)$ for each SAM. In the

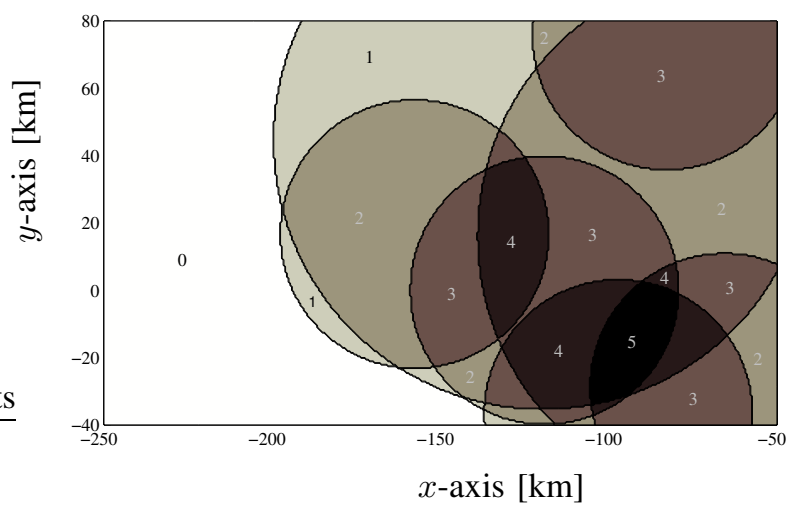

Fig. 6. Number of SAMs within firing range

scenario shown in Figure 5, some points are within firing range of up to five SAMs (cf. Figure 6).

Figure 7 shows the partition of the airspace described above for a group of 1 through 3 UCAVs flying above the scenario in Figure 5. For this figure it was assumed that each UCAV would jam at most one SAM. A conclusion to be drawn from this figure is that a small number of UCAVs flying in close formation and using jamming in a cooperative fashion can safely penetrate a heavily defended region. Note that with only 3 UCAVs the perimeter defended by the seven SAMs has been broken.

\section{MINIMUM-RISK PATH-PLANNING}

Given a region $\mathcal{R} \subset \mathbb{R}^{3}$ populated by multiple SAM sites, minimum-risk path planning refers to the computation of a path $\rho:[0, T] \rightarrow \mathcal{R}$ for the group of $m$ UCAVs that starts at an initial position $x_{i}$ and ends at a final position $x_{f}$, maximizing the probability that the UCAVs will not be tracked by any of the SAMs' radars and will therefore survive the journey. It was shown in [1] that minimumrisk path planning can be reduced to a weighted anisotropic shortest-path problem. Here, we extend the formulation in [1] to consider cooperative jamming.

The probability that at least one of the UCAVs in the group will be tracked by the $j$ th SAM in an elementary time interval of length $d t$ is given by

$$
\eta_{j}\left(x, \dot{x}, z_{j}\right) d t
$$

where $x$ and $\dot{x}$ denote the position and velocity of the group of UCAVs, and $z_{j}$ the position of the $j$ th SAM. The function $\eta_{j}$ is called the tracking density with respect to the jth SAM. In the region $\mathcal{R}_{\text {green }}$ defined in Section III, $\eta_{j}(\cdot)$ is always equal to zero whereas in the region $\mathcal{R}_{\text {red }}$ it is always equal to one. In the regions $\mathcal{R}_{\text {orange }}$ and $\mathcal{R}_{\text {yellow }}$, the value of $\eta_{j}(\cdot)$ depends on the elevation and azimuth angles $\theta_{\mathrm{el}}, \theta_{\mathrm{az}}$, which are a function of the relative position $z_{i}-x$ of the SAM with respect to the UCAVs, as well as the UCAVs' direction of motion $\dot{x}$. In particular, $\eta_{j}(\cdot)$ will be equal to zero as long as $\sigma_{\text {burn }}^{j}(R)$ is larger than $\sigma_{i}^{j}\left(\theta_{\mathrm{el}}, \theta_{\mathrm{az}}\right), \forall i$ and equal to one otherwise. The index $i$ ranges over the UCAVs in the group.

As shown in [1], the risk model (7) leads to a probability that no UCAV will be tracked at any point in the path $\rho$ given by

$$
p_{\text {survive }}[\rho]=e^{-\int_{0}^{T} \ell(\rho(t), \dot{\rho}(t)) d t},
$$

where $\ell(x, v):=\sum_{j=1}^{n} \eta_{j}\left(x, v, z_{j}\right)$. Since the function $s \mapsto e^{-s}$ is monotone decreasing, paths that are optimal (maximal) with respect to the reward $p_{\text {survive }}[\rho]$ are also optimal (minimal) with respect to the cost

$$
J[\rho]:=\int_{0}^{T} \ell(\rho(t), \dot{\rho}(t)) d t .
$$

We can therefore compute these paths by solving the weighted anisotropic shortest-path problem considered in [1]. The path generated by this optimization would serve as a reference trajectory for the group, to be used by algorithms such as the ones proposed in [7-9]. One could also take fuel consumption and/or path length as constraints.

Figure 8 shows two minimum-risk paths for a group of five UCAVs flying over the region in Figure 5. The two paths start at the same given point (top-left) and are allowed to end anywhere with the target area represented by a circle (top middle). For the path corresponding to the solid line it was assumed that the UCAVs do not have jamming capabilities and simply attempt to minimize their overall RCS as in [1]. The UCAVs utilize a "zigzag" path to explore the small RCS that they expose to a radar located almost directly in front or behind them (cf. Figure 2). The dotted line path was obtained assuming that the UCAVs have jamming capabilities. This path falls almost exclusively in the region $\mathcal{R}_{\text {green }}$, which permits an almost straight-line path with much lower risk. All optimizations were done numerically using the algorithms described in [1].

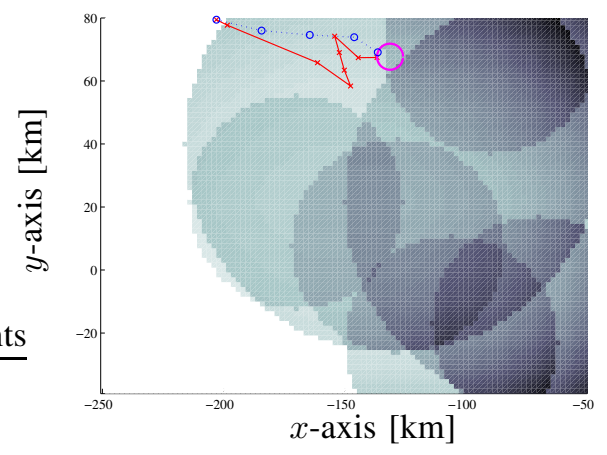

Fig. 8. Minimum-risk path with (dashed) and without (solid) Jamming 

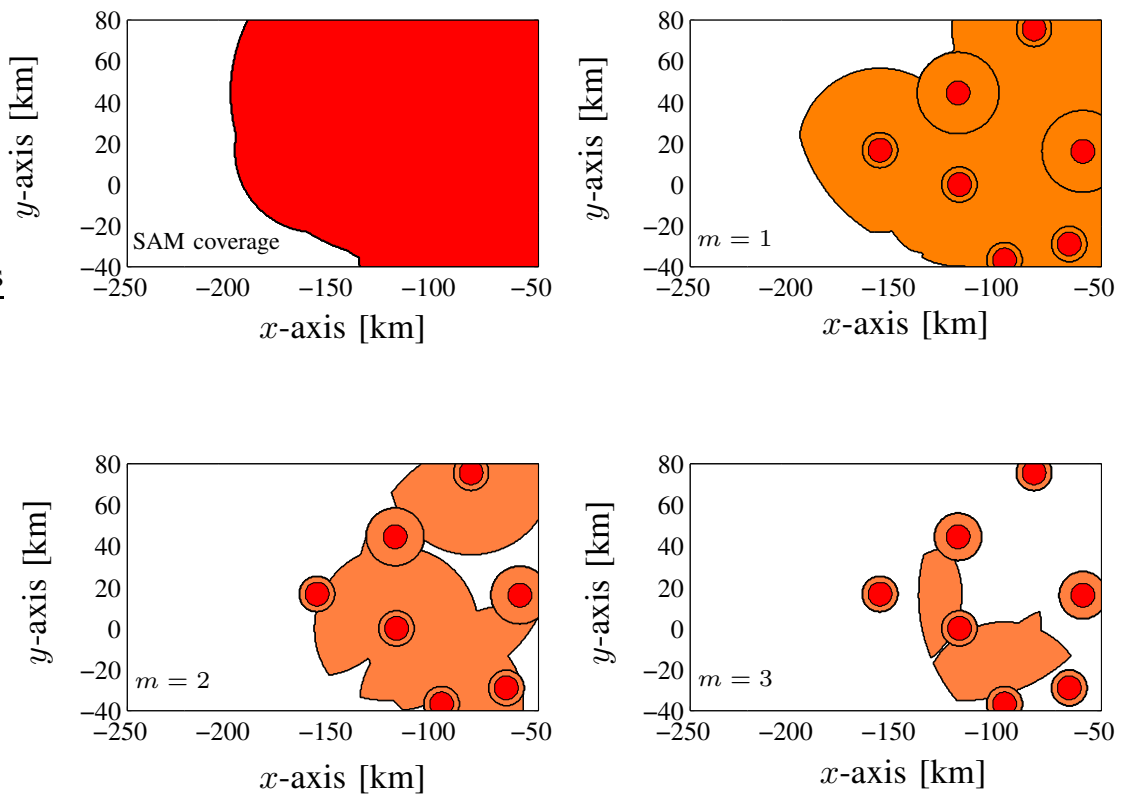

Fig. 7. Partition of the airspace for a group of $m$ UCAVs flying above the scenario in Figure 5 . The top-left figure simply shows the area within firing range of at least one SAM. The remaining figures show $\mathcal{R}_{\text {yellow }} \cup \mathcal{R}_{\text {green }}$ in white, which essentially corresponds to the region where the group of UCAVs can fly in any direction (azimuth), provided that the elevation is appropriately selected. The small darker circles correspond to $\mathcal{R}_{\text {red }}$ and the remaining solid area to $\mathcal{R}_{\text {orange. }}$. All plots represent projections of the three-dimensional regions on the plane.

The paths obtained were validated through simulation in the Open Experimental Platform (OEP) developed by Boeing for the DARPA/IXO program Mixed Initiative Control of Automa-teams (MICA) [2,3] and resulted in essentially no destruction of UCAVs when there was a feasible path that could avoid $\mathcal{R}_{\text {yellow }}$ and $\mathcal{R}_{\text {red }}$, or at least not remain in these areas for more than a few seconds. Although this simulator is not realistic from a dynamic simulation perspective, it still enforces kinematic constraints (such as maximum turn and ascent rates) that are consistent with realistic models of UCAVs. Moreover, it simulates the bank angle during turns and its impact on the RCS of the aircraft with respect to tracking radars.

Remark 2: In reality, the tracking density $\eta_{j}(\cdot)$ is not exactly always zero or one. It will be very low (but positive) when $\sigma_{\text {burn }}^{j}(R)$ is larger than all the $\sigma_{i}^{j}\left(\theta_{\mathrm{el}}, \theta_{\mathrm{az}}\right)$ and almost one otherwise. The MICA challenge problem [2] provides specific values for $\eta_{j}(\cdot)$ as a function of the RCSs $\sigma_{i}^{j}\left(\theta_{\mathrm{el}}, \theta_{\mathrm{az}}\right)$. These were used to compute the paths in Figure 8, but a binary approximation does not lead to significantly different results.

\section{CONClusion}

We considered a scenario where a group of Unmanned Combat Air Vehicles (UCAVs) flies in a close formation and cooperates in their use of jamming resources to (i) superimpose their signals to increase the range over which jamming is effective against a particular radar, and/or (ii) direct their transmitters to different radars to conceal the formation from multiple radars. The impact of jamming on minimum-risk path planning was also investigated and we proposed a methodology for minimum-risk path planning for autonomous UCAVs capable of jamming. Our results were validated through simulation in Boeing's OEP.

Current research includes the study of jamming in scenarios with an Integrated Air Defense System (IADS), in which multiple radars cooperate to track aircraft.

\section{REFERENCES}

[1] J. Kim and J. P. Hespanha, "Discrete approximations to continuous shortest-path: Application to minimum-risk path planning for groups of UAVs," in Proc. of the 42nd Conf. on Decision and Contr., Dec. 2003.

[2] "Challenge problem version 1.3, MICA OEP software version 1.3," 2003, DARPA/IXO Program "Mixed Initiative Control for Automateams.".

[3] User's Guide, MICA OEP Software Version 1.3, Boeing, 2003, DARPA/IXO Program "Mixed Initiative Control for Automa-teams.".

[4] "Electronic warfare and radar systems engineering handbook," Avionics Department of the Naval Air Warfare Center Weapons Divisio, Point Mugu, CA, Tech. Rep. TP-8347, Oct. 2003. [Online]. Available: https://ewhdbks.mugu.navy.mil/contents

[5] J. P. Hespanha and H. H. Kizılocak, "Efficient computation of dynamic probabilistic maps," in Proc. of the 10th Mediterranean Conf. on Contr. and Automat., July 2002.

[6] J. P. Hespanha, H. H. Kızılocak, and Y. S. Ateşkan, "Probabilistic map building for aircraft-tracking radars," in Proc. of the 2001 Amer. Contr. Conf., June 2001.

[7] J. A. Fax and R. M. Murray, "Information flow and cooperative control of vehicle formations," in Proc. of the 15th World Congress of Int. Federation of Automat. Contr., July 2002.

[8] P. Ogren, E. Fiorelli, and N. E. Leonard, "Formations with a mission: Stable coordination of vehicle group maneuvers," in Proc. of the Int. Symp. on the Mathematical Theory of Networks and Syst., Aug. 2002

[9] A. Pant, P. Seiler, and K. Hedrick, "Mesh stability of look-ahead interconnected systems," IEEE Trans. on Automat. Contr., vol. 47, no. 2, pp. 403-407, Feb. 2002. 